\title{
Using settling behaviour to study mesostructural organization of cement pastes and superplasticizer efficiency
}

\author{
Caroline Autier ${ }^{\mathrm{a}}$, Nathalie Azéma ${ }^{\mathrm{a}, *}$, Pascal Boustingorry ${ }^{\mathrm{b}}$ \\ a Ecole des Mines d'Alès, Centre des matériaux, 6 avenue de Clavières, 30319 Alès cedex, France \\ ${ }^{\mathrm{b}}$ CHRYSO, 7 rue de l'Europe, 45300 Sermaises, France
}

\section{H I G H L I G H T S}

- Highlighting influence of organomineral interactions on dispersion state of cement suspension.

- Characterization of different polycarboxylate efficiency by settling behaviour study.

- Quantification of stability ranges in function of molecular structure of PCE by a Phase Separation Index (PSI).
G R A P H I C A L A B S T R A C T

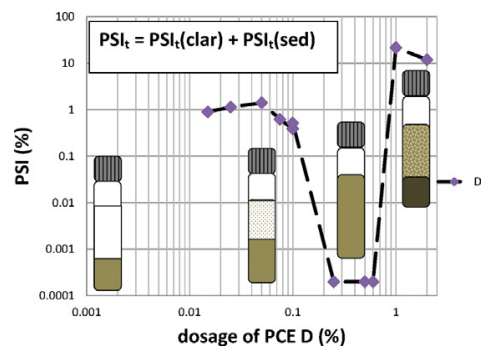

\section{A B S T R A C T}

Superplasticizers are polymer admixtures which have been used for decades in the building industry. Their role is to increase the workability of fresh concrete by dispersing cement particles through an electrostatic or steric repulsion mechanism. Their efficiency is generally related to their adsorption ability and intrinsic rheological behaviour of mineral materials. However, at an intermediate scale, mesostructural organization is a major parameter that controls not only the fluidity but also the homogeneity and the stability of a suspension. The aim of this paper is to highlight the relationship between mesostructure and organo-mineral interactions occurring between superplasticizers and mineral particles. The influence of the molecular structure of polycarboxylates (PCEs) on the destabilization phenomena was characterized. Stability ranges were assessed using Turbiscan MA 2000 and quantified by a Phase Separation Index (PSI) inspired by previous works. A relationship with mesostructural organization was established by correlation with the dispersion state characterized by laser granulometry. The affinity of PCEs with cement particles as a function of molecular structure was characterized by Total Organic Carbon (TOC) measurements.

Keywords:

Stability

Dispersion state

Adsorption

Superplasticizers

Cement paste

\section{Introduction}

Superplasticizers have been developed for decades in order to increase the workability of fresh concretes by dispersion of mineral particles composing concrete pastes. Many studies deal with the influence of the molecular structure of superplasticizers on their

\footnotetext{
* Corresponding author. Tel.: +33 0466785356; fax: +33 0466785365.

E-mail address: nathalie.azema@mines-ales.fr (N. Azéma).
}

efficiency [1-5]. The influence of the cement characteristics like chemical composition or specific surface as well as mixing procedure was also studied [6,7]. Most studies in this field focus on adsorption property of superplasticizers and rheological behaviour of the materials in which they are incorporated [8-11]. In this way, fluidity is directly related to the affinity of the polymer for cement surfaces.

However, at an intermediate scale, mesostructural organization is a major parameter that controls not only the fluidity but also the homogeneity and the stability of a suspension. 
Table 1

Characteristics of the cement used.

\begin{tabular}{|c|c|c|}
\hline Chemical characteristics & & Value \\
\hline $\mathrm{Na}_{2} \mathrm{O}$ equivalent & & $0.35 \%$ \\
\hline Oxides & $\%$ & \\
\hline $\mathrm{Al}_{2} \mathrm{O}_{3}$ & 4.7 & \\
\hline $\mathrm{Fe}_{2} \mathrm{O}_{3}$ & 2.8 & \\
\hline $\mathrm{SiO}_{2}$ & 19.4 & \\
\hline $\mathrm{CaO}$ & 65.8 & \\
\hline $\mathrm{SO}_{3}$ & 5.1 & \\
\hline Surface characteristics & & Value \\
\hline B.E.T. specific surface area & & $1.1 \mathrm{~m}^{2} / \mathrm{g}$ \\
\hline Blaine specific surface & & $0.4 \mathrm{~m}^{2} / \mathrm{g}$ \\
\hline \multicolumn{3}{|c|}{ Specific surface area by granulometry } \\
\hline - Deionized water & & $0.3 \mathrm{~m}^{2} / \mathrm{g}$ \\
\hline - Dry way & & $0.4 \mathrm{~m}^{2} / \mathrm{g}$ \\
\hline \multicolumn{3}{|l|}{ Dormant period } \\
\hline - By conductivity $\left(25^{\circ} \mathrm{C}\right)$ & & $3 \mathrm{~h} 45$ \\
\hline
\end{tabular}

Mesostructural organization can be defined as the organization resulting of interactions between unit or agglomerated particles in an intermediate scale between unit particle one and the suspension. This relationship was studied by some authors with various mineral suspensions like kaolins or alumina. While some studies focused on the influence of the addition of dispersants or surface treatments on particles, others focused on the influence of the suspension solid volume fraction [12-20]. For this reason, the Turbiscan MA2000 from the Formulaction Company was used. This device was indeed developed to study nascent instability phenomena in concentrated suspensions allowing to take into account interparticle interactions present in the suspension $[21,22]$. The coupling of this technique with other ones such as SEM or laser granulometry allows to provide information about mesostructural organization and hence interparticle interactions.

The aim of this paper is to highlight the influence of mesostructural organization, in connection with organo-mineral interactions (occurring between the superplasticizer and cement particles) on the homogeneity and the stability of fresh cement pastes. This study is focused on Ordinary Portland Cement (OPC) pastes during the dormant period when the chemical reactivity slows down. In these conditions, the physicochemical phenomena are favoured over hydration reactions.

The stability and homogeneity of cement pastes over time as a function of the dosage of several PCE-based superplasticizers (PCE) were characterized using Turbiscan MA 2000 and laser granulometry. The influence of the molecular structure of the PCE on the destabilization phenomena and the stability range was highlighted. A Phase Separation Index (PSI) inspired by the works of Vié [12] was defined to characterize the stability of the suspensions. The relationship with mesostructural organization was established through the correlation with the dispersion state characterized by laser granulometry.

The correlation with adsorption by Total Organic Carbon (TOC) measurements allowed showing the influence of organo-mineral interactions on larger scales. Indeed, the affinity of a PCE with the cement particles as a function its molecular structure was highlighted.

\section{Materials and methods}

\subsection{Materials}

A commercial Ordinary Portland Cement provided by Calcia, the characteristics of which are presented in Table 1 , was chosen for the

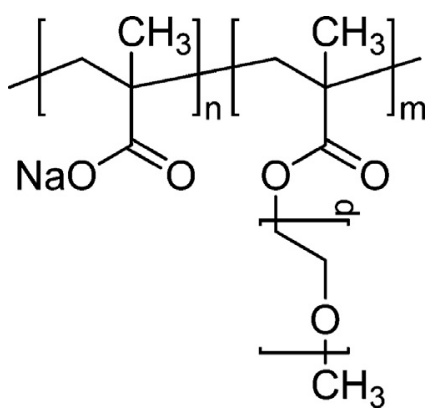

Fig. 1. Molecular structure of the PCEs tested.

preparation of the paste. The cement composition was determined using X-ray fluorescence. The $\mathrm{Na}_{2} \mathrm{O}$ equiv. is low, so competitive adsorption with sulphate ion is low [5]. The dormant period, determined by conductivity and calcium concentration measurement, lasts $3 \mathrm{~h} 45$ at $25^{\circ} \mathrm{C}$ [23]. The Blaine fineness and specific surface areas by laser granulometry show similar low values and slightly higher values than the B.E.T. specific surface area (factor 3), which characterizes the absence of porosity and few agglomeration phenomena on the dry powder.

Four PCEs provided by the CHRYSO Company (France) were studied and called A, B, C and D later in this paper. They are composed of a poly(methacrylic acid) backbone, responsible for adsorption, partially esterified with methoxy-terminated polyoxyethylene side-chains (PEO) responsible for steric repulsion. The chemical structure is shown in Fig. 1. The ester grafting ratio $(m / n+m)$ increases from 10 to $40 \%$ through polymers A to D with a fixed PEO length $(p)$ of 45 monomers. This series of polymers was chosen in order to study the influence of grafting ratio on the efficiency of PCE.

\subsection{Pastes preparation}

Cement suspensions were prepared by adding $200 \mathrm{~g}$ of cement powder to admixture solutions in deionized water (liquid chosen to control the water quality: $\mathrm{pH}$, ionic conductivity, etc.) with water to cement ratio of 0.5 (including the amount of water provided by the admixture). Each polycarboxylate was tested through a dosage range up to $1 \%$ of dry active matter with respect to cement weight. The mixing equipment consisted in a twisted mixing anchor blade adapted to a Stuart, SS 30 mixer and a stainless steel beaker. The suspension was mixed at a speed of $185 \mathrm{rpm}$ during $20 \mathrm{~min}$ before analysis that must be done during the $2 \mathrm{~h}$ of the dormant period (checked by conductivity and calcium concentration measurements).

For granulometric, suspension stability and Total Organic Carbon measurements, paste samples were first diluted in deionized water. This perturbation should modify ionic strength and solidsolution equilibrium of studied system, but it was neglected in a first approximation. As far as possible the dilution was the same (30\% weight) for suspension stability and TOC analysis.

\subsection{Characterization of the cement pastes by TOC and laser granulometry}

The particle size distributions of the cement pastes were measured in deionized water using a LS 230 laser granulometer (Beckman Coulter). The optical model used was computed with a refractive index with a real part of 1.7 and an imaginary part of 1 . The analysis time was fixed to $1 \mathrm{~min}$. The particle size distributions obtained were expressed in a percent by volume and were normalized to $100 \%$. The particle size distributions as a function of 
the height in the column were performed after 40 min of settling of the diluted paste.

The PCE adsorption was measured according to the depletion method. The quantity of consumed polymer was estimated by difference between the quantity of PCE introduced in the paste and the measured quantity of PCE remaining in pore solution. For that purpose, suspensions were diluted and let to settle in the same conditions as for the settling measurement. Then, the liquid phase at the top of the column was extracted and the pore solution was separated from the particles by filtration with a $0.2 \mu \mathrm{m}$ membrane filter and neutralized with concentrated hydrochloric acid. The quantity of remaining PCE was also determined by Total Organic Carbon (TOC) analysis using a commercial TOC-VCPN analyser (Shimadzu, Japan).

In the same way, solutions of PCE with the same dosages as in the pastes were analyzed and used as an initial concentration measurement. In order to eliminate the amount of organic carbon released by the cement (coming from grinding aids) the value of TOC of the unadmixtured paste was considered as background and subtracted from all measurements.

\subsection{Multiple light scattering analysis by Turbiscan MA 2000}

\subsubsection{Theoretical basis}

The Turbiscan MA 2000 analyser from the Formulaction Company is designed to detect faster than naked eye nascent instability phenomena in concentrated dispersions (e.g. suspensions and emulsions). The ability to work in concentrated media (up to $20 \%$ volume to volume for suspensions) allows taking into account the interparticle interactions that may occur in the dispersion.

The principle of this technique is based on the detection of the light flux transmitted and backscattered as a function of the height in the dispersion column. The device is composed of a detection head which moves vertically with a step of $40 \mu \mathrm{m}$ along a cylindrical cell (15 mm diameter and $110 \mathrm{~mm}$ height) containing the sample. Two measurements at $0^{\circ}$ and $135^{\circ}$ from the incident beam $(\lambda=850 \mathrm{~nm})$ direction are performed and correspond respectively to the intensity of transmitted and backscattered light.

As shown in Eqs. (1) and (2), there is a relationship between the backscattered light intensity and the mesostructural parameters such as particle volume fraction and mean diameter $[21,22]$.

$\mathrm{BS} \approx \frac{1}{\sqrt{\lambda^{*}}}$

$\lambda *(\Phi, d)=\frac{2 d}{3 \Phi(1-g) Q_{s}}$

where BS is the intensity of the backscattered light (\%); $\lambda^{*}$ is the photon transport length; $d$ is the mean diameter of the particles; $\Phi$ is the solid volume fraction; $g$ is the asymmetry factor; and $Q_{S}$ is the scattering efficiency factor.

The last two parameters are optical parameters from the Lorentz-Mie Theory.

Similarly, Eqs. (3) and (4) present another relationship between the percentage of transmitted light $\left(T_{r}\right)$ and these parameters.

$T_{r} \approx \exp \left[\frac{-r_{i}}{\lambda}\right]$

$\lambda=\lambda *(1-g)=\frac{2 d}{3 \Phi Q_{s}}$

where $r_{i}$ is the internal radius of the measurement cell.

By evolution of the acquired profiles as a function of time it is then possible to detect particle size changes (through dispersion or agglomeration) or particle migration (sedimentation) as a function of the height in the sedimentation column.

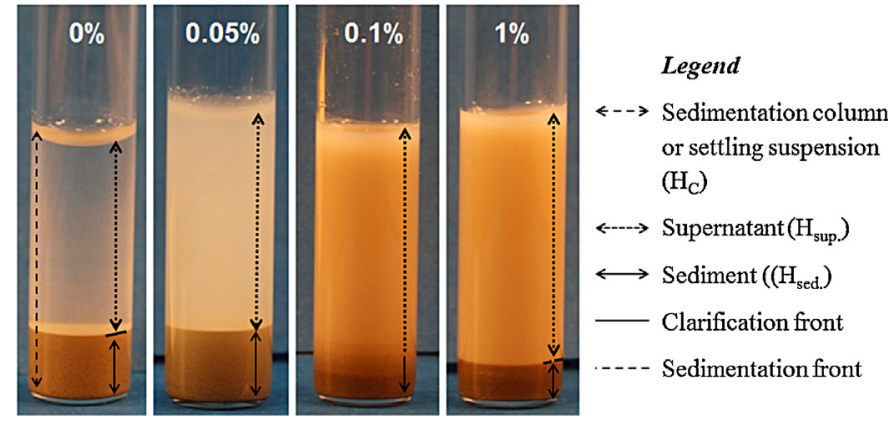

Fig. 2. Visual observations of sedimentation columns in function of dosage of $P C E$ after $40 \mathrm{~min}$ of settling.

\subsection{Measurement parameters}

The stability of the cement suspensions was studied at room temperature $\left(25^{\circ} \mathrm{C}\right)$ using a Turbiscan MA 2000. The analysis time lasted $40 \mathrm{~min}$ and the time elapsed between two profiles was $2 \mathrm{~min}$.

Before analysis, the paste was previously diluted at 30\% weight in deionized water and submitted to a moderate magnetic stirring during about a minute. After dilution, the solid volume fraction is $12 \%$.

\section{Results and discussion}

\subsection{Visual observations and granulometric characterizations of sedimentation columns}

As a preliminary step, visual observations of some sedimentation columns allowed to describe settling behaviours as a function of the dosage of PCE incorporated (Fig. 2). These kinds of destabilization phenomena are called clarification, relating to concentrated suspensions [24-26]. In all columns two phases can be distinguished at 40 min of settling: a supernatant at the top and sediment at the bottom. Their formation kinetics and characteristics seem to be influenced by the PCE dosage. Indeed, two regimes are observed: at low dosages of PCE (0-0.05 wt.\% with respect to cement weight) the supernatant is clear and separated from the sediment (which does not seem to reach its maximum packing yet) with a sharp boundary called "clarification front". Note that in the column containing $0.05 \%$ of PCE, the supernatant is hazier than in the unadmixtured column suggesting some colloidal particles dispersion by the PCE. Furthermore, the particle size distributions taken in the bulk of the sediment area, presented in Fig. 3a, are similar. This result shows the homogeneity of the sediment.

At higher dosages of PCE (0.1-1 wt.\% with respect to cement weight) the supernatant is opaque and seems heterogeneous. This is confirmed by Fig. 3b which presents a gradient of size in the sedimentation column at $0.1 \%$ of PCE. Note that the sediment at $0.1 \%$ is thinner than the $0.05 \%$ one, and the sedimentation front is difficult to observe.

In order to differentiate these two cases of settling, the second one presenting an opaque supernatant will be called "sediment formation". The front delimiting an opaque supernatant and packed sediment will be called "sedimentation front". Let us point out that all these distinctions are made for analysis times of $40 \mathrm{~min}$, when the settling may not be completely over.

These first observations allowed us to highlight two destabilization phenomena depending on the dosage of PCE within the conditions of duration and solid volume fraction used in the analysis: a destabilization phenomenon at low dosages related to the clarification of a supernatant, and another at high dosages related to the packed sediment formation. 

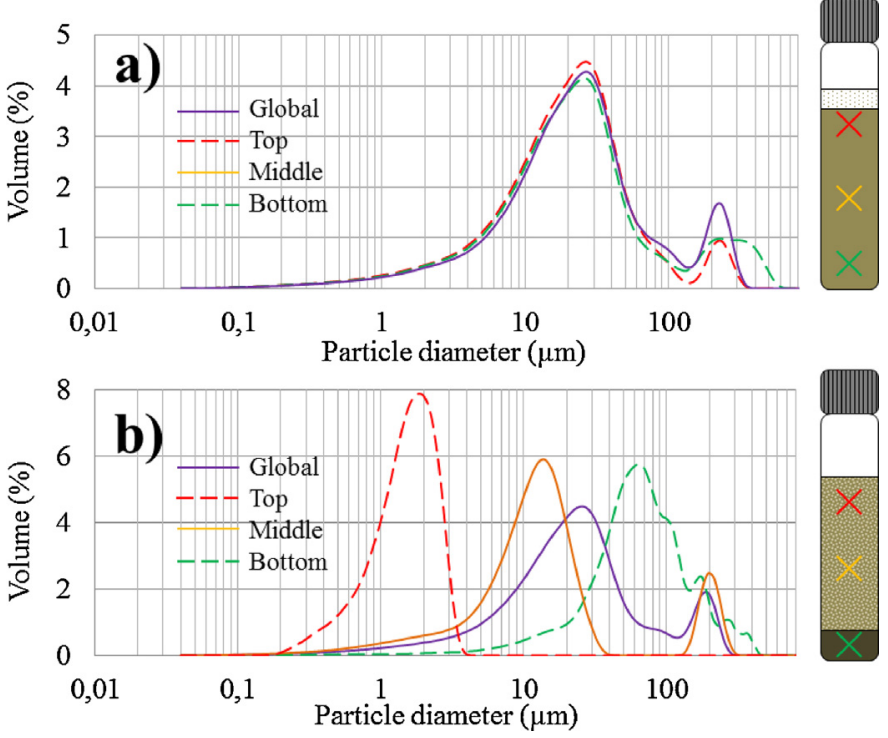

Fig. 3. Particle size distributions at several heights in the column with (a) $0.05 \%$; (b) $0.1 \%$ of PCE C.

In order to develop a quantitative approach, a settling study was performed using the Turbiscan MA 2000 transmission or backscattering data. The influence of the molecular structure and the dosage of PCE on the suspension stability were also investigated as discussed in the next section.

\subsection{Study of instability phenomena by Turbiscan MA 2000}

\subsubsection{Supernatant evolution using the transmission profiles}

At low dosages, the transmission profiles resulting from the light passing through the suspension is used to characterize the clarification of the supernatant thus the influence of the PCE addition on suspension stability. As shown in Fig. 4 (case a) where the height of the sedimentation column is represented on the abscise axe, a clear supernatant is identified by a high value of transmission percentage up to $50-60 \%$. In this case, two parameters may then characterize the clarification of the supernatant: the displacement of the clarification front and the transmission rate time evolution. Indeed, the

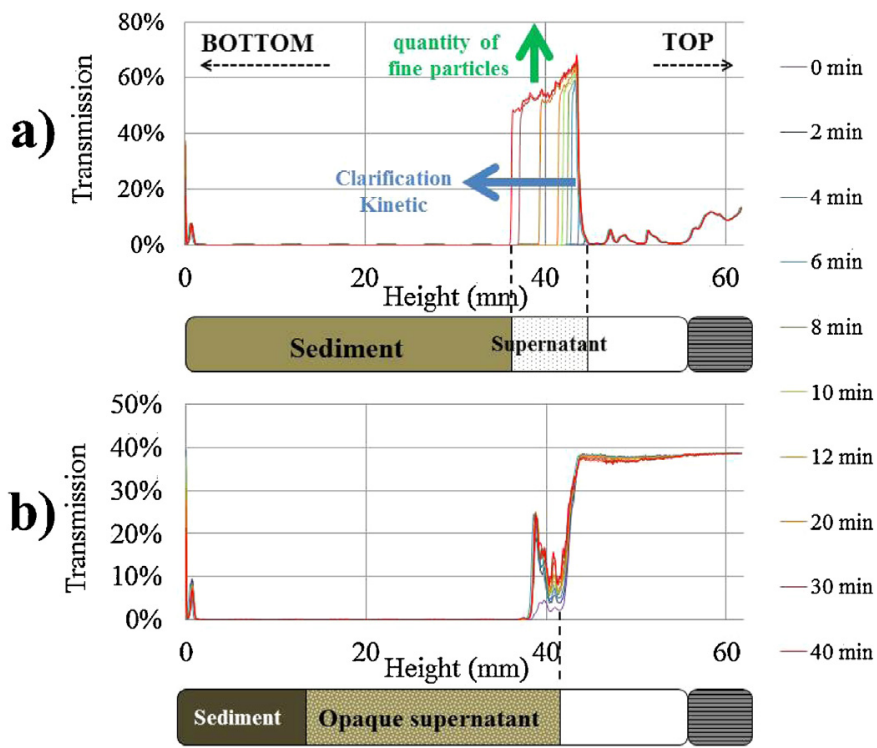

Fig. 4. Transmitted light of diluted pastes admixtured with (a) $0.05 \%$; (b) $0.1 \%$ of PCE C. release of unsettling particles by the PCE resulting from deagglomeration of (sub)micronic particles or hydrate precipitates [27] may accordingly decrease the amount of transmitted light. However, when the supernatant is too opaque there is no transmitted light (case $b$ ) and the transmission profiles cannot be used.

Note that a stabilization of the suspension translates into a decrease of the displacement of clarification front and a high value of transmission percentage in the supernatant.

\section{(a) Evolution of the clarification front}

As a first step, the evolution of the supernatant relative thickness as a function of the dosage of the PCEs tested is presented in Fig. 5. In all cases, a variable threshold time is observed indicating a delay before the onset of the clarification process. Later on, the evolution of the curve is linear up to a plateau corresponding to a significant slowdown of the phenomenon. Two parameters may then be extracted: the clarification threshold time and the kinetic of clarification corresponding to the initial slope of the curve.

The PCEs A and D present opposite behaviours; when the dosage of PCE A increases, the value of threshold time increases up to $25 \mathrm{~min}$ (at $0.1 \%$ of PCE) and the slope decreases continuously from 0.35 to $0 \mathrm{~min}^{-1}$ (at $0.25 \%$ of PCE), traducing a continuous slowdown of the clarification front evolution with dosage. In the case of the PCE D, both parameters present constant values whatever the dosage (the threshold time remaining constant at about $5 \mathrm{~min}$ and the slope at about $0.35 \mathrm{~min}^{-1}$ ). The PCE D does not seem to influence the clarification front evolution up to $0.25 \mathrm{wt}$.\% above which there is no clarification anymore. The PCEs B and C present intermediate behaviours. Like the PCE D, they do not seem to affect settling up to a critical dosage (respectively $0.075 \%$ and $0.05 \%$ ) beyond which, they abruptly present a PCE A-like regime, where threshold time increases and slope decreases when dosage increases.

\section{(b) Turbidity of the supernatant}

In order to quantify and compare turbidity of supernatants influenced by the presence of dispersed particles and/or precipitated hydrates, the evolution of light transmission in the supernatant as a function of the dosage of PCE was monitored over time. Note that this parameter is not widely used in the literature. The results are presented in Fig. 6.

Much like the previously observed trends, all curves present a threshold time corresponding to the delay before the clarification starts, the evolution of which depends on the molecular structure of the PCE. Then, the transmission signal increases up to a variable plateau where it remains steady until the end of the analysis time. According to Eqs. (3) and (4), this value depends on the mean diameter and solid volume fraction of unsettling particles remaining in the supernatant.

The previously observed slowdown of the supernatant relative thickness with the dosage of PCE A does not seem to be correlated to the percentage of transmitted light which remains at about $60 \%$ up to a dosage of $0.075 \%$. This value, close to the pure water value (about 90\%), corresponds to a very clear supernatant.

On the opposite, the increase of the PCE D dosage leads to a stable transmission values up to a dosage of $0.08 \%$ (at about $40 \%$ transmission which corresponds to a turbid supernatant). Then, these values decrease until there is no transmission (opaque supernatant at $0.25 \%$ ).

When increasing the dosage of PCEs B and $C$ the evolution of the transmission rate seems correlated to the supernatant relative thickness. 

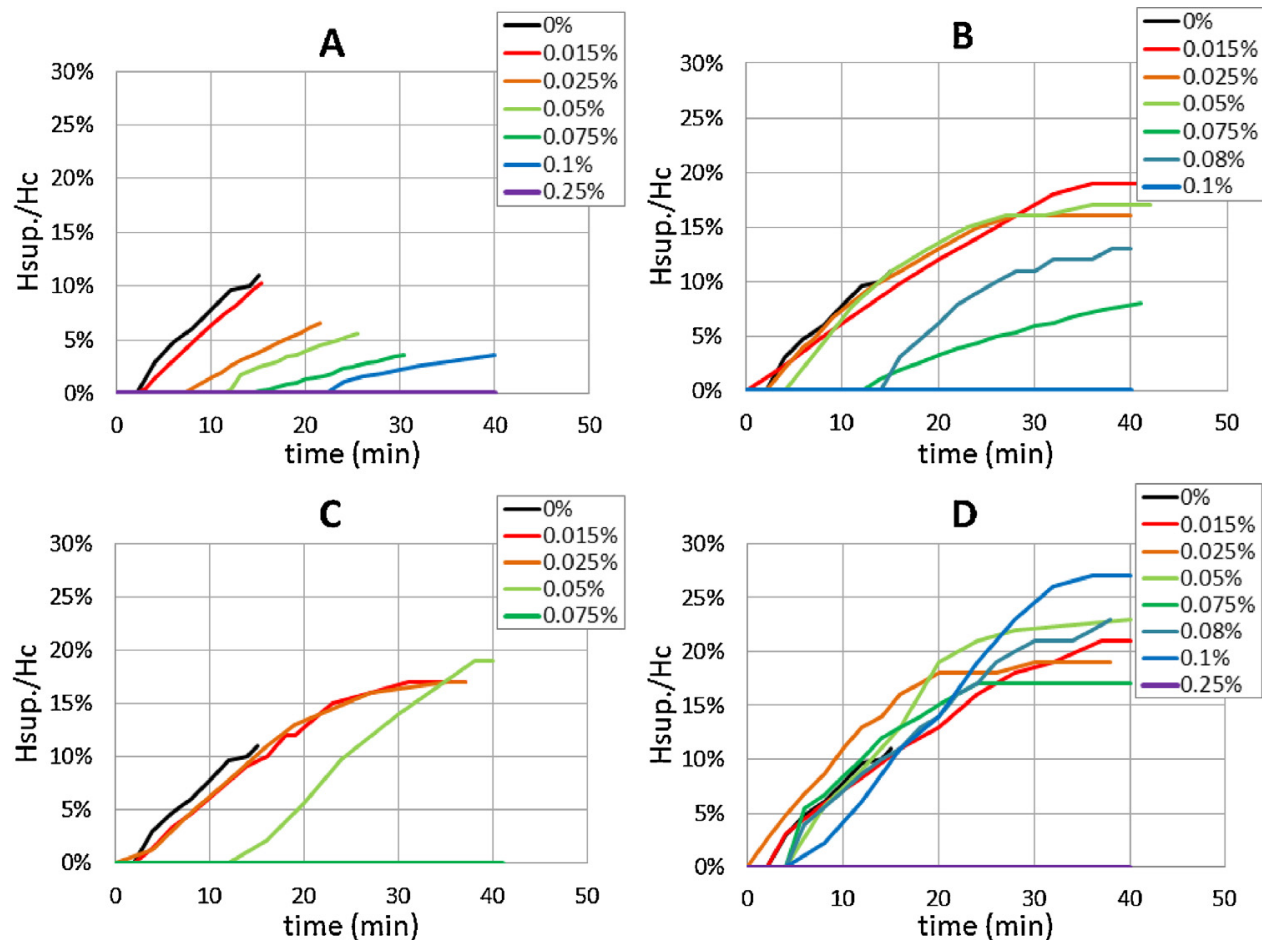

Fig. 5. Evolution of the relative supernatant thickness as a function of the dosage of PCEs A, B, C, D.

To sum up our observations about the influence of the molecular structure of PCE, two opposite mechanisms define a range of behaviours:

- For low grafting ratio polymer, a gradual increase of clarification threshold time with the increase of PCE dosage associated to a thin clear supernatant $\left(H_{\text {sup. }} / H_{C}<10 \%\right)$.
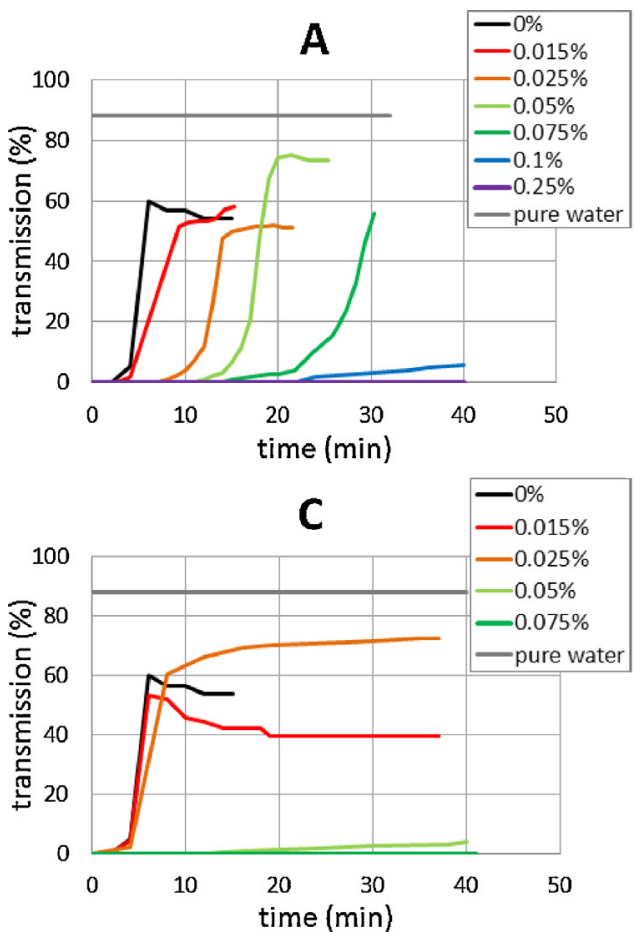

- For highest grafting ratio polymer, on the opposite, a short constant clarification threshold time associated to an abundant cloudy supernatant.

The two intermediate polymers simply feature a combination of those extremes, following the grafting ratio value.

(c) Comparison with mesostructural organization and PCE adsorption
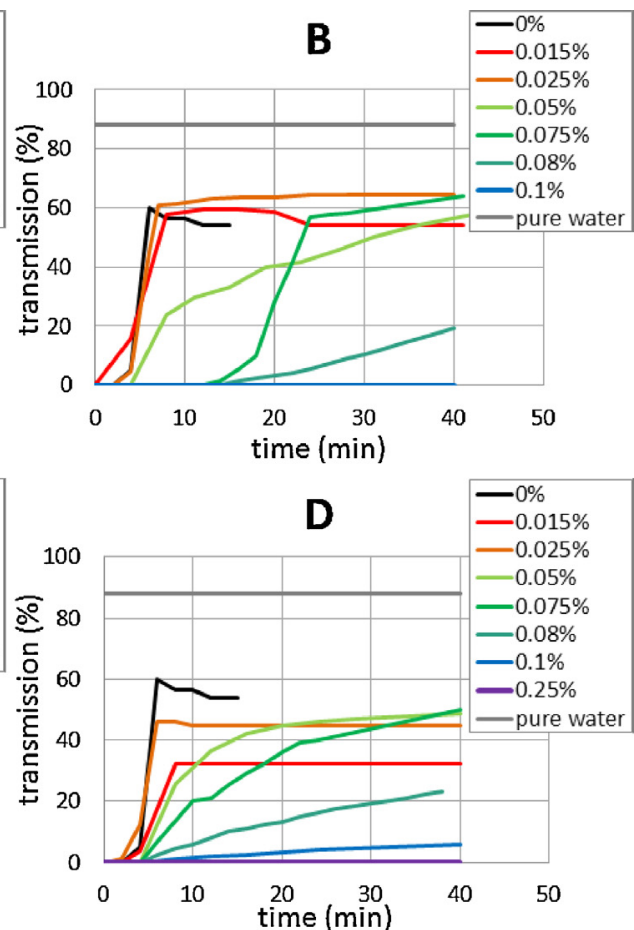

Fig. 6. Evolution of the percentage of transmission over time function of the dosage of PCEs A, B, C, D. 


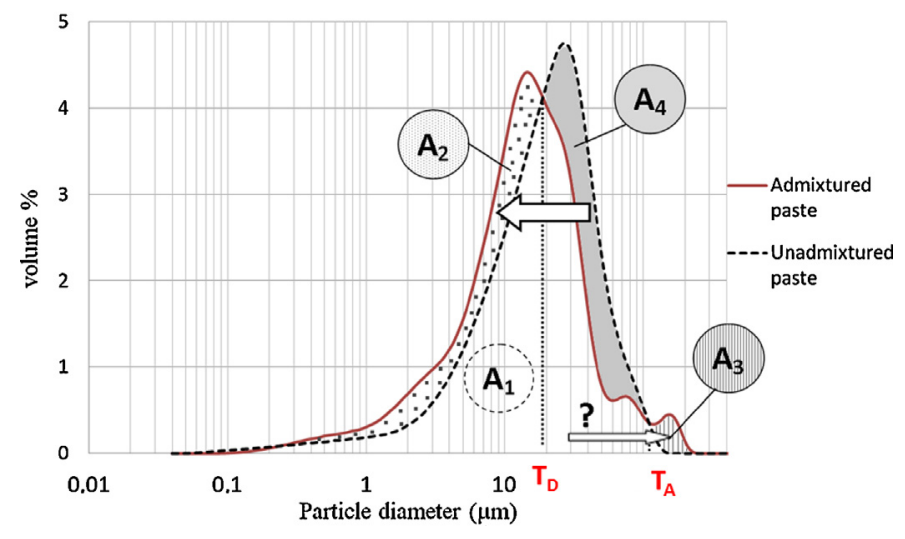

Fig. 7. Evolution of the particle size distribution by addition of superplasticizer [26].

Mesostructural organization was characterized by computing a dispersion index defined in a previous study [27]. This index is based on the comparison of the particle size distributions of a unadmixtured reference paste with an admixtured paste. As shown in Fig. 7, the addition of superplasticizer causes a shift of the particle size distribution towards smaller diameters, defining intersection points, $T_{\mathrm{D}}$ and $T_{\mathrm{A}}$ (T for particle transfer, $\mathrm{D}$ for dispersion and $\mathrm{A}$ for agglomeration), which represent points of matter transfer between agglomerated and more dispersed populations.

The dispersion index is defined as follows:

$I_{\mathrm{D}}(\%)=\left(\frac{\mathrm{As}_{<\mathrm{TD}}-\mathrm{Aref}_{<\mathrm{TD}}}{A_{1}}\right) \times 100=\left(\frac{A_{2}}{A_{1}}\right) \times 100=A_{2}$

where

- As $<$ TD is the area lower than $T_{\mathrm{D}}$ located under the curve of the sample, Aref $_{<\mathrm{TD}}$ is the area lower than $T_{\mathrm{D}}$ located under the curve of the reference, $A_{1}$ is the total area located under the curve of the reference ( $=100 \%$ because the curves are normalized), $A_{2}$ is the area located between the curve of the sample and the curve of the reference under the particle transfer point $T_{\mathrm{D}}$.

The evolution of the dispersion index as a function of the dosage of PCE is presented in Fig. 8.

The influence of the PCE A on the dispersion state of the paste clearly stands out. More precisely when its dosage increases, dispersion state of the paste seems to increase in the studied dosage range (an optimal dispersion state is not yet reached). Oppositely, the other PCEs present an optimal dispersion state at $0.08 \mathrm{wt} . \%$ and appear to be efficient molecules. These results may be compared to the stability behaviours previously highlighted. They show an

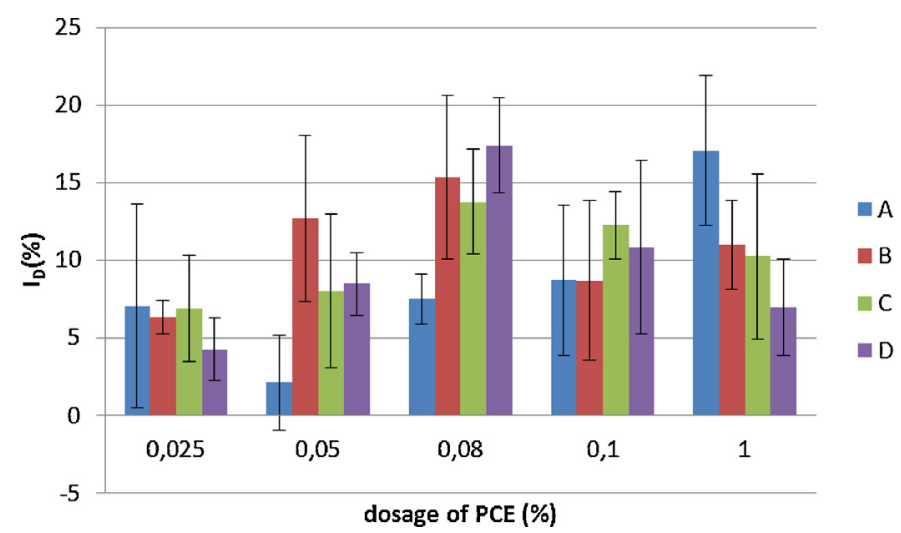

Fig. 8. Evolution of the dispersion index as a function of the dosage of PCEs A, B, C, D.

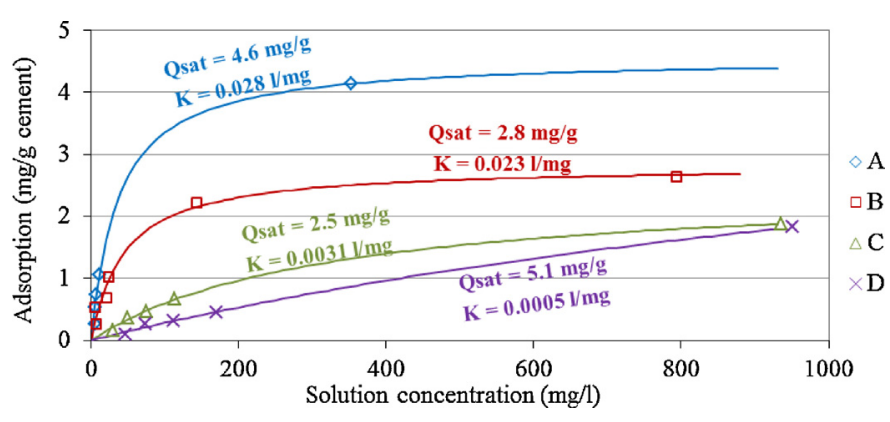

Fig. 9. Langmuir isotherms of the PCEs tested.

optimum of stabilization at respectively $0.1 \%$ and $0.075 \%$ for the PCEs $\mathrm{B}$ and $\mathrm{C}$ according to the dosage of optimal dispersion state.

In the case of PCE D, stabilization presents a shift towards higher dosages when compared to PCEs B and C (at 0.25\% - cf. Fig. 6), independently of an optimum dispersion state observed at 0.08 wt.\% by laser granulometry study. These differences of results may originate from the agitation of sample during the particle size distribution measurement which may affect interparticular bonds and lead to the same dispersion state for PCEs B, C and D.

In order to quantify the maximum adsorption and affinity of the PCEs with cement surface in these analysis conditions (cf. point 2.2), the Langmuir isotherm was applied to PCE consumption measured by TOC analysis. This model, presented in Eq. (6), is based on three assumptions: monolayer coverage, adsorption site equivalence and independence.

$Q=Q_{\text {sat }} \times \frac{K C}{1+K C}$

where $Q$ is the amount adsorbed $(\mathrm{mg} / \mathrm{g}) ; Q_{\text {sat }}$ is the maximum adsorbed $(\mathrm{mg} / \mathrm{g}) ; K$ (adsorption/desorption ratio) is a constant that characterize the affinity of the polymer with the surface $(1 / \mathrm{mg})$; $C$ is the concentration remaining in solution $(\mathrm{mg} / \mathrm{l})$.

$Q_{\text {sat }}$ and $K$ values are obtained by Langmuir linearization i.e. by noticing that $Q=Q_{\text {sat }}(1+K C-1) /(1+K C)=Q_{\text {sat }}-Q_{\text {sat }} /$ $(1+K C)=Q_{\text {sat }}-1 / K(Q / C)$. Hence, Langmuir parameters may be determined by measuring the intercept and the negative reciprocal of the slope of the $Q=f(Q / C)$ curve. The Langmuir isotherms of the PCEs tested are presented in Fig. 9.

In all cases, PCE adsorption increases with dosage up to a saturation plateau. The adsorption affinity somewhat measured by $K$ increases with carboxylate group ratio in the molecular structure $[6,10]$. According to the isotherm, the PCE D does not seem to have reached the maximal adsorption (plateau) in the range explored whereas the others seem to have. As a general rule the maximum adsorption decreases when the grafting ratio increases which highlights the increasing self-hindrance induced by increasing POE amount.

The low adsorption of the PCE D on cement particles allows explaining its weak influence on the suspension stability up to $0.25 \%$. On the opposite, although the PCE A is adsorbed in higher amounts, its relatively lower efficiency on the dispersion state correlates to its low efficiency on the stability which is probably due to its low grafting ratio leading to a poor steric repulsion. The PCEs B and C having intermediate grafting ratios present an intermediate adsorptive behaviour. Indeed, they seem to have a sufficient carboxylate ratio to adsorb onto cement and a sufficient ester grafting ratio to disperse efficiently. This optimized molecular structure allows stabilizing the suspension even at lower dosages. This optimum effect in the molecular structure is well known in the literature [3-5]. 


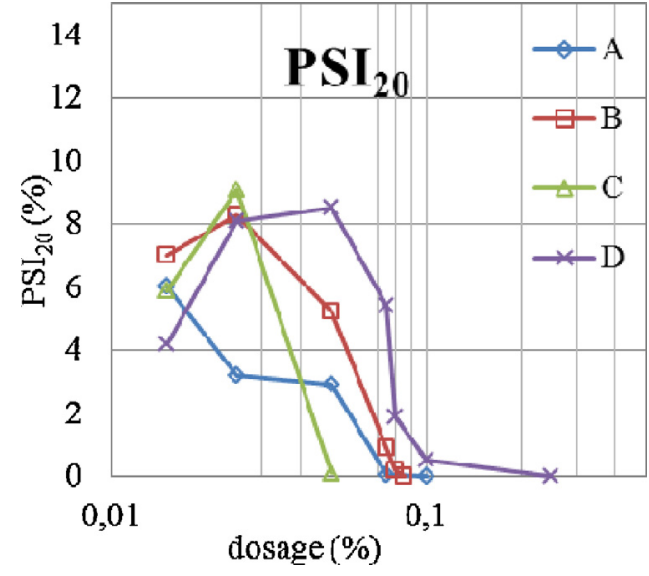

Fig. 10. Evolution of the $\mathrm{PSI}_{20}$ (clar.) as a function of the dosage of PCEs A, B, C, D.

\section{(d) Definition of a Phase Separation Index characterizing the clarifica- tion phenomenon}

A Phase Separation Index, denoted PSI(clar.) is defined to quantify this instability phenomenon, instability increasing with the index value. This index combines supernatant volume and light transmission rate in order to characterize all clarification phenomena at a given settling time (20 min was chosen later in the work) according to Eq. (7):

$\operatorname{PSI}_{\mathrm{t}}($ clar. $)=\frac{H_{\text {sup }}}{H_{\mathrm{c}}} \times \% \operatorname{Tr} .($ supernatant $)$

where $H_{\text {sup. }} / H_{\mathrm{c}}$ represents the volume proportion corresponding to the clarification volume and \% $\operatorname{Tr}$.(supernatant) is related to the quantity and size of suspended particles in the supernatant. A stable suspension is defined by a $\mathrm{PSI}_{t}$ (clar.) value equal to zero $(\% \mathrm{Tr}=0$ and a minimum value of $H_{\text {sup. }}$.). Note that in the literature, the transmission percentage is seldom used.

Fig. 10 shows the evolution of the $\mathrm{PSI}_{20}$ (Clar.) as a function of the dosage of the PCEs studied. Except for the lowest dosages, the PSI decreases continuously when PCE dosage (thus adsorption) increases as a result of the dispersing effect.

The suspension stability with PCE C appears at a lower dosage than the others $(0.05 \mathrm{wt} . \%)$. While for PCE D stability occurs at a higher dosage (0.25 wt.\%).

\subsubsection{Sediment formation using the backscattering profiles}

In order to obtain information on the stability of opaque settling suspensions, $135^{\circ}$-backscattered light profiles were also processed. In this study, the profile time evolution is used to investigate the influence of PCE addition on the homogeneity and stability of opaque supernatants as well as the formation of sediments formed at high dosages. As shown in Fig. 11, stable and homogeneous opaque settling suspensions present steady backscattering values (about 40\%) over time and column height (case a). So, there is no observable evolution of the particle size or volume fraction in the suspension during the analysis.

In the case of unstable suspensions (case b), two phenomena may be observed.

The first one located in the opaque supernatant area corresponds to the increase of the mean value of backscattered light over time. According to Eq. (1) presented in Section 2.4.1, this may be related to a decrease of mean particle size or/and a local increase of the particle volume fraction. A variation of the mean particle size is assumed here since the particle volume fraction is relatively low (12\%) and its influence is therefore less significant [22].

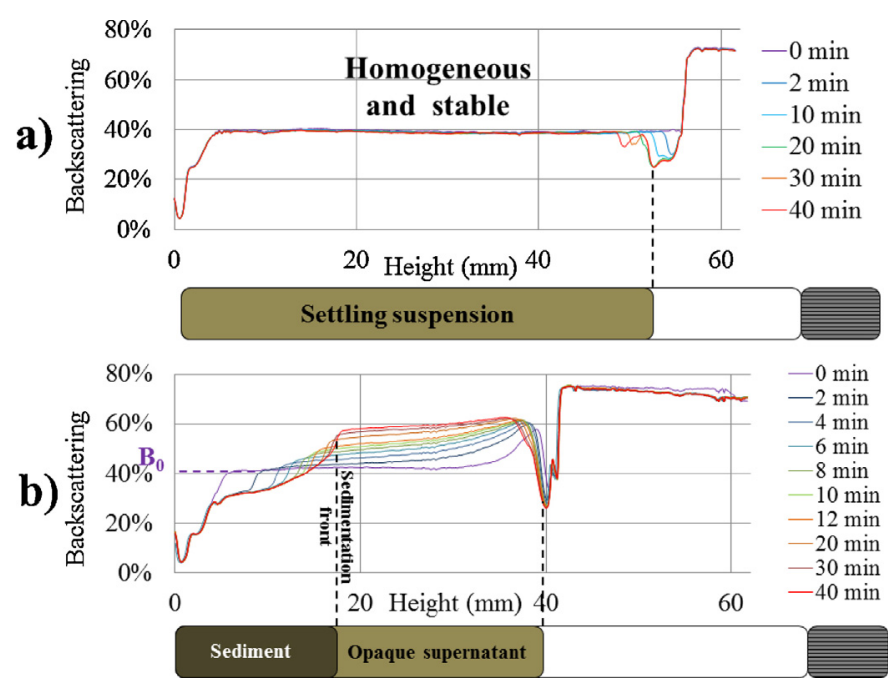

Fig. 11. Backscattered light in (a) homogeneous and stable settling suspension (0.05\% PCE A) and (b) heterogeneous and unstable settling suspension (0.5\% PCE A).

The second phenomenon corresponds to the formation of sediment characterized by two parameters: its relative thickness and the mean backscattering rate (the first $5 \mathrm{~mm}$ at the bottom of the analysis cell should be discarded since they correspond to a distortion induced by the vial glass bottom).

In a first step, the time evolution of the mean value of backscattered light in the opaque supernatant divided by the initial backscattering rate is presented in Fig. 12. Its evolution seems to depend neither on the dosage nor on the structure of the PCE. Indeed, almost all curves fall on a master curve and $\mathrm{BS} / \mathrm{BS}_{0}$ reaches an asymptotic value of about 1.4 after $40 \mathrm{~min}$. Therefore, the time evolution of mean diameter and volume fraction in this area seems to depend neither on the dosage nor on the molecular structure of the PCE. The increase of the $\mathrm{BS} / \mathrm{BS}_{0}$ during the first 20 min corresponds to a decrease of the mean particle diameter induced by the settling of larger particles and probably by a dispersion phenomenon in these opaque suspensions.

In a second step, parameters characterizing the sediment formation were studied at $20 \mathrm{~min}$ when the phenomenon previously described has reached a steady state. Their evolutions are presented in Fig. 13.

Suspensions enter the sediment formation regime from critical dosages which are lower in the case of the PCEs B and C: respectively $0.12 \%$ and 0.08 wt.\% whereas they are respectively $0.25 \%$ and $0.35 \%$ in the cases of the PCEs A and D. However, the sediment formation kinetics and the backscattering rate in the sediment

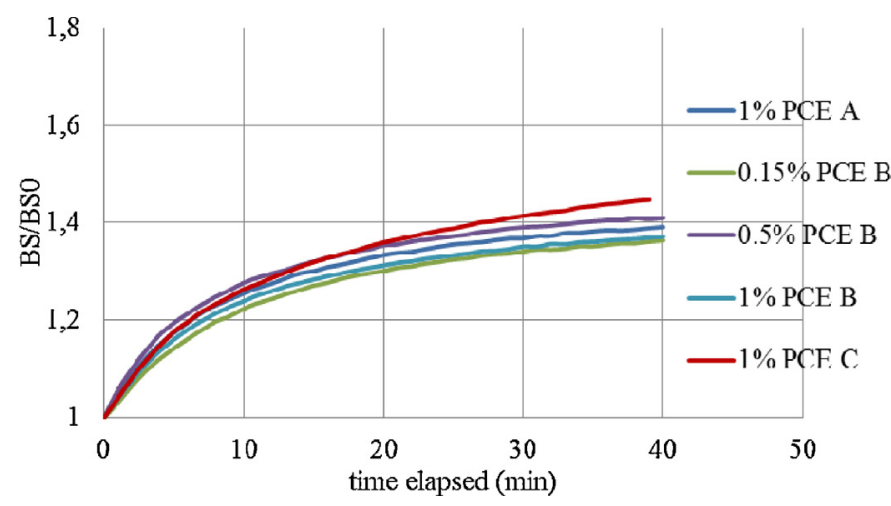

Fig. 12. Evolution of some relative backscattering in opaque supernatants over time. 

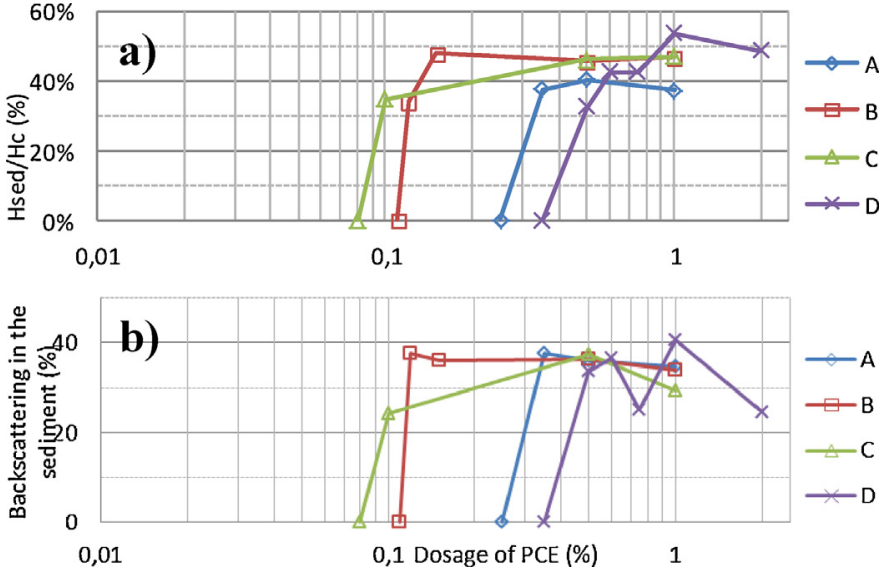

Fig. 13. Evolution of (a) relative height of sediment; (b) relative BS value of sediment, at $20 \mathrm{~min}$, as a function of the dosage of PCEs A, B, C, D.

seem unaffected by the dosage and the molecular structure of the PCE (for all these formulations $H_{\text {sed }} / H_{\mathrm{c}}$ is about 40-50\%). Similarly, the value of the backscattering rate is approximately equal in all characterized sediments (30-40\%).

The sediment formation regime is then characterized by critical dosages being a non monotonous function of the grafting ratio but neither the settling kinetics nor the compacity of the sediment are affected.

Under these conditions, the Phase Dispersion Index presented in Eq. (4) and defined by Vié et al. in a previous study should be constant [12]. This index integrating the latter parameters is defined as follows and its dosage dependence at $20 \mathrm{~min}$ is presented in Fig. 14:

$\operatorname{PSI}_{\mathrm{t}}($ sed. $)=\frac{H_{\text {sed. }}}{H_{\mathrm{c}}} \times \% \mathrm{BS}($ sed. $)$

Note that in the same way as for the clarification phenomenon, the molecular structure of PCE influences the onset of the instability phenomenon at high dosage. The PCEs B and C present similar trends as they induce sediment formation at lower dosages (respectively 0.12 and $0.08 \mathrm{wt} . \%)$ than the two others $(0.25 \%$ for A and $0.35 \%$ for D). In comparison with dispersion indices displayed in Fig. 8, the dispersion indices with PCEs B and C present no evolution between $0.1 \%$ and $1 \%$ which explains why no change in the $\mathrm{PSI}_{20}$ (sed.) is observed. In the case of the PCE D, the increase of the dispersion index in this range explains the increase of the $\mathrm{PSI}_{20}$ (sed.). In the case of PCE A, although dispersion degree increases, stability decreases in this range.
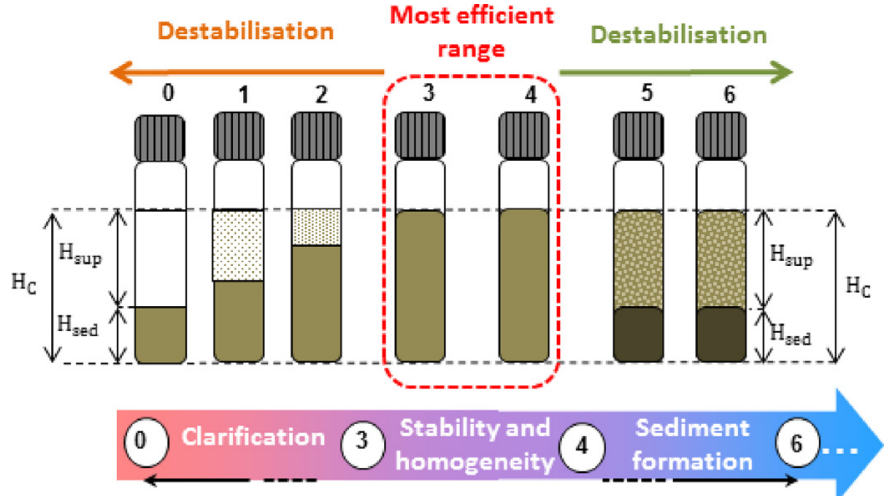

Fig. 15. Schematic representation of the destabilization phenomena as a function of the dosage of PCE.

\subsubsection{Most efficient dosage ranges as a function of the molecular structure of the PCE}

As shown previously and schematized in Fig. 15, the two destabilization phenomena occur at different dosage ranges and do not correspond to the same physicochemical phenomenon. All PCEs tested present same trend: first, they tend to stabilize the clarification phenomenon when dosage increases up to a most efficient range corresponding to the optimal stabilization of the suspension. The width of the most efficient range of dosage also depends on the molecular structure of the PCE. Beyond this regime, a second step of destabilization occurs with the sediment formation. The latter phenomenon is independent on the molecular structure and the PCE dosage.

In order to quantify the variations of stability of cement pastes as a function of the dosage and molecular structure of PCE, a global Phase Separation Index, defined as the sum of the previous PSIs, is defined:

$\mathrm{PSI}_{\mathrm{t}}=\mathrm{PSI}_{\mathrm{t}}$ (clar.) $+\operatorname{PSI}_{\mathrm{t}}($ sed. $)$

The PSIs evolutions as a function of PCEs dosage are presented in Fig. 16. The PCEs B and C which have intermediate ester grafting ratios show similar values and induce narrower stability ranges than the other PCEs at lower dosages. The PCE A with the lowest grafting ratio confers a wider stability range at higher dosages. Then PCE D induces the widest stability range but for higher dosages.

From these observations it may be inferred that the molecular structure of the PCE influences the width and the dosage limits of the stability range through the interplay between the adsorbed amount and the intrinsic steric repulsion. The non-monotonic

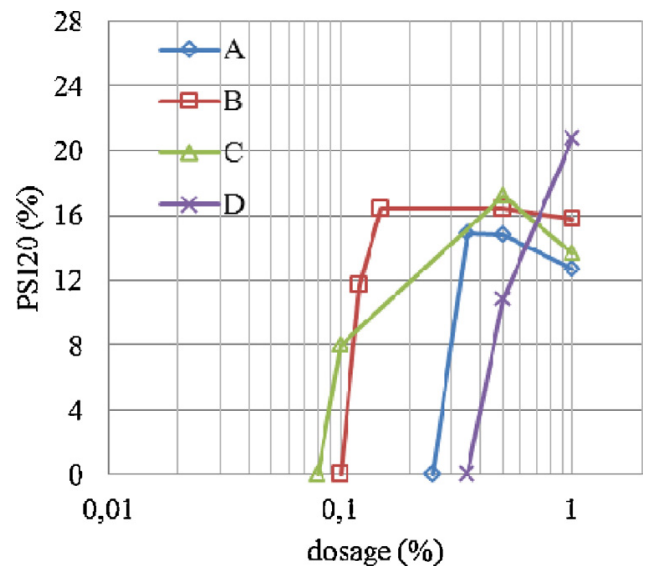

Fig. 14. $\mathrm{PSI}_{20}$ (sed.) as a function of the dosage of PCEs A, B, C, D.

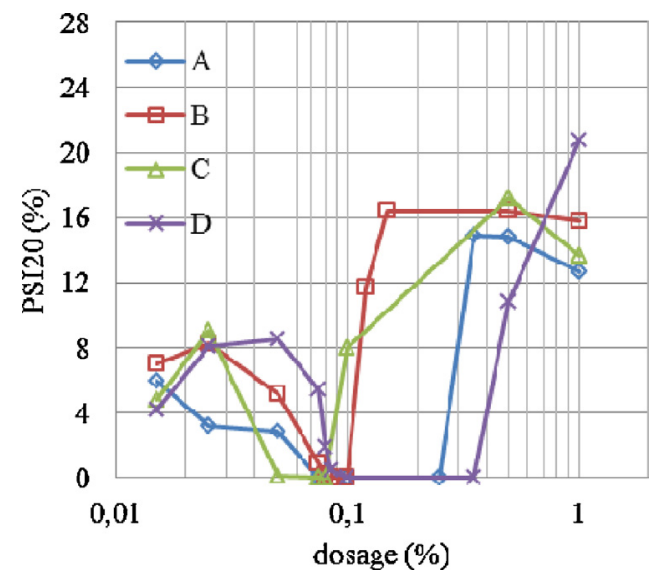

Fig. 16. Evolution of the $\mathrm{PSI}_{20}$ as a function of the dosage of PCEs A, B, C, D. 
evolution of the PSI as a function of the ester grafting ratio highlights an optimum effect of the molecular structure of the PCE. This non-monotonous effect of the structure may be explained by the following reasons.

According to Flatt et al. [28] adsorbed comb polymers take a conformation described as a chain of hemispheric blobs the thickness of which is given by the blob radius:

$R_{\mathrm{AC}} \approx P^{7 / 10} N^{-1 / 10}$

where $P$ is the graft length expressed in monomer number (constant in our study) and $N$ is the number of backbone repeating units containing one graft, i.e. the reciprocal of the grafting ratio.

The adsorbed layer thickness, taken as an estimate of the range of steric repulsive forces, is then expected to increase as the grafting ratio raised to the power of $1 / 10$.

On the opposite it was shown that increasing the grafting ratio at a constant graft length decreases the affinity for the substrate $[6,10]$, which we confirm in our study (see the $K$ values in Fig. 9).

We conclude that grafting ratio has an opposite influence on the affinity on the one hand and on the steric repulsion range on the other hand.

At low grafting ratios the polymer features then a high affinity for the cement surface but the grafts are too few along the backbone to form a sufficiently thick repulsive layer. Thus it requires a high adsorbed amount of polymer (i.e. a high dosage) to reach a density of grafts on the surface sufficient for triggering sedimentation. This is the case of PCE A with high-dosage sedimentation transitions.

On the opposite, at high grafting ratios each polymer forms a thicker repulsive object but the affinity is so weak that it requires high dosages before obtaining a sufficient surface coverage. PCE $D$ seems to behave this way with once again high-dosage sedimentation transitions. PCEs A and D would feature then a similar behaviour (high transition dosages), but for very different reasons.

Between these two extremes there exists a domain where affinity and layer thickness are balanced in such a way that dosage efficiency is maximum, which seems to be the case for PCEs B and $\mathrm{C}$ as they show the lowest transition dosages.

\section{Conclusions}

The relationship between organo-mineral interaction, mesostructural organization, stability and homogeneity of suspensions was highlighted. For that purpose, the stability was characterized using Turbiscan MA 2000. Two destabilization regimes were highlighted: the first one occurring at lower dosages consists in a clarification of the supernatant which tends to disappear with increasing the dosage of PCE. The suspension then enters an intermediate stable dispersion state. The second destabilization corresponds to the sediment formation regime which consists in a packing of the sediment occurring at even higher dosages. A Phase Separation Index (PSI) taking into account these two destabilization phenomena was defined in order to quantify the stability of suspensions. This index was compared with the dispersion index characterizing the dispersion state of particles and with the adsorption isotherms. In this way, the macroscopic behaviour was related to mesostructural organization of the paste. The influence of PCE structure and affinity with mineral surface on the dispersion state was also highlighted.

More precisely, as the grafting ratio increases, adsorption affinity decreases and intrinsic steric repulsion increases. At a low grafting ratio, adsorption is quite high for a given dosage, but the adsorbed layer features too weak a repulsion efficiency (PCE A case). At a high grafting ratio the adsorbed layer is thicker but adsorption affinity is too weak (PCE D case). There exists an intermediate regime where steric repulsion and adsorption are more evenly balanced so that dispersion efficiency is maximal.

\section{Acknowledgement}

The authors wish to thank Mr. Claude VALENTIN (Ciments Calcia - Italcementi Group) for having provide the cements used in the project.

\section{References}

[1] K. Yamada, Effects of the chemical structure on the properties of polycarboxylate-type superplasticizer, Cement and Concrete Research 30 (2) (2000) 197-207

[2] C. Li, N. Feng, Y. Li, R. Chen, Effects of polyethlene oxide chains on the performance of polycarboxylate-type water-reducers, Cement and Concrete Research 35 (5) (2005) 867-873

[3] J.-Y. Shin, J.-S. Hong, J.-K. Suh, Y.-S. Lee, Effects of polycarboxylate-type superplasticizer on fluidity and hydration behavior of cement paste, Korean Journal of Chemical Engineering 25 (6) (2008) 1553-1561.

[4] A. Zingg, F. Winnefeld, L. Holzer, J. Pakusch, S. Becker, L. Gauckler, Adsorption of polyelectrolytes and its influence on the rheology, zeta potential, and microstructure of various cement and hydrate phases, Journal of Colloid and Interface Science 323 (2) (2008) 301-312

[5] K. Yamada, Controlling of the adsorption and dispersing force of polycarboxylate-type superplasticizer by sulfate ion concentration in aqueous phase, Cement and Concrete Research 31 (3) (2001) 375-383.

[6] A. Zingg, F. Winnefeld, L. Holzer, J. Pakusch, S. Becker, R. Figi, L. Gauckler, Interaction of polycarboxylate-based superplasticizers with cements containing different C3A amounts, Cement and Concrete Composites 31 (3) (2009) $153-162$.

[7] R. Flatt, A simplified view on chemical effects perturbing the action of superplasticizers, Cement and Concrete Research 31 (8)(2001) 1169-1176.

[8] J. Plank, C. Hirsch, Impact of zeta potential of early cement hydration phases on superplasticizer adsorption, Cement and Concrete Research 37 (4) (2007) 537-542.

[9] S. Srinivasan, S.A. Barbhuiya, D. Charan, S.P. Pandey, Characterising cement-superplasticizer interaction using zeta potential measurements, Construction and Building Materials 24 (12) (2010) 2517-2521.

[10] A. Zingg, F. Winnefeld, L. Holzer, J. Pakusch, S. Becker, R. Figi, L. Gauckler, Interaction of polycarboxylate-based superplasticizer with cement containing different C3A amounts, Cement and Concrete Composites 31 (3) (2009) 153-162.

[11] M. Palacios, Y.F. Houst, P. Bowen, F. Puertas, Adsorption of superplasticizer admixtures on alkali-activated slag pastes, Cement and Concrete Research 39 (8) (2009) 670-677.

[12] R. Vié, N. Azéma, J.C. Quantin, E. Touraud, M. Fouletier, Study of suspension settling: a approach to determine suspension classification and particle interactions, Colloids and Surfaces A 298 (2007) 192-200.

[13] N. Azéma, Sedimentation behavior study by three optical methods - granulometric and electrophoresis measurements, dispersion optical analyser, Powder Technology 165 (2006) 133-139.

[14] A. Lafaurie, N. Azéma, L. Ferry, J.-M. Lopez-Cuesta, Powder Technology 192 (2009) 92-98

[15] O. Burgos-Montes, R. Moreno, Stability of concentrated suspensions of $\mathrm{Al}_{2} \mathrm{O}_{3}-\mathrm{SiO}_{2}$ measured by multiple light scattering, Journal of the European Ceramic Society 29 (2009) 603-610.

[16] E.H. Lee, M.K. Lee, C.K. Rhee, Preparation of stable dispersions of Ni nanoparticles using a polymeric dispersant in water, Materials Science and Engineering A $449-451$ (2007) 765-768.

[17] D. Dudašová, G.R. Flåten, J. Sjöblom, G. Øye, Study of asphaltenes adsorption onto different minerals and clays. Part 2. Particle characterization and suspension stability, Colloids and Surfaces A 335 (2009) 62-72.

[18] A. Amiri, G. Øye, J. Sjöblom, Influence of pH, high salinity and particle concentration on stability and rheological properties of aqueous suspensions of fumed silica, Colloids and Surfaces A 349 (1-3) (2009) 43-54.

[19] M. Balastre, J.F. Argillier, C. Allain, A. Foissy, Role of polyelectrolyte dispersant in the settling behaviour of barium sulphate suspension, Colloids and Surfaces A 211 (2002) 145-156.

[20] M. Wiśniewska, Influences of polyacrylic acid adsorption and temperature on the alumina suspension stability, Powder Technology 198 (2010) 258-2696.

[21] O. Mengual, TURBISCAN MA 2000: multiple light scattering measurement for concentrated emulsion and suspension instability analysis, Talanta 50 (2) (1999) 445-456.

[22] O. Mengual, G. Meunier, I. Cayre, K. Puech, P. Snabre, Characterisation of instability of concentrated dispersions by a new optical analyser: the TURBISCAN MA 1000, Colloids and Surfaces A 152 (1-2) (1999) 111-123.

[23] S. Maximilien, Study of the reactivity of clinkers by means of the conductometric test, Cement and Concrete Research 27 (1) (1997) 63-73.

[24] D. Quemada, Energy of interaction in colloids and its implications in rheological modeling, Advances in Colloid and Interface Science 98 (1) (2002) 51-85. 
[25] T.F. Tadros, Correlation of viscoelastic properties of stable and flocculated suspensions with their interparticle interactions, Advances in Colloid and Interface Science 68 (1996) 97-200.

[26] P. Coussot, C. Ancey, Rhéophysique des pâtes et des suspensions, L'Editeur: EDP Sciences, 1999, pp. 264.
[27] C. Autier, N. Azéma, J.M. Taulemesse, L. Clerc, Mesostructure evolution of cement pastes with addition of superplasticizers highlighted by dispersion indices, Powder Technology 249 (2013) 282-289.

[28] R.J. Flatt, I. Schober, E. Raphael, C. Plassard, E. Lesniewska, Conformation of adsorbed comb copolymer dispersants, Langmuir 25 (2009) 845-855. 